\title{
MHD flow past a nonlinear stretching/shrinking sheet in carbon nanotubes: stability analysis
}

\begin{abstract}
This work aims at evaluating the effects of magnetohydrodynamics (MHD) on the stagnation point flow along a nonlinear stretching/shrinking sheet in carbon nanotubes. Numerical methods for ordinary differential equations are obtained using the BVP4C solver in MATLAB software. Two kinds of base fluids, particularly water and kerosene, with singlewalled and multi-walled carbon nanotubes are adopted in this analysis. The effect of various limitations on the Nusselt number and skin friction coefficient, as well as the temperature and velocity profiles are examined. From the numerical results, it is observed that non-unique solutions are visible for some limits of shrinking parameter. It is also found that nonlinear parameter and magnetohydrodynamic parameter act in widening the range of solution to exist. Therefore, the stability of flow was executed to identify the most stable solution between these two solutions. The stable and unstable flow of the first and second solutions, respectively, are confirmed.
\end{abstract}

Keyword: Carbon nanotube; Nonlinear stretching/shrinking sheet; Magnetohydrodynamic; Stability analysis 